\title{
Two-Phase Optimal Guidance Law considering Impact Angle Constraint with Bearings-Only Measurements
}

\author{
Tianning Wang, Shengjing Tang, Jie Guo, and Haoqiang Zhang \\ School of Aerospace Engineering, Beijing Institute of Technology, Beijing 100081, China \\ Correspondence should be addressed to Shengjing Tang; tangsj@bit.edu.cn
}

Received 8 March 2017; Revised 9 July 2017; Accepted 16 July 2017; Published 15 August 2017

Academic Editor: Christopher J. Damaren

Copyright (C) 2017 Tianning Wang et al. This is an open access article distributed under the Creative Commons Attribution License, which permits unrestricted use, distribution, and reproduction in any medium, provided the original work is properly cited.

\begin{abstract}
The implementation of advanced guidance laws with bearings-only measurements requires estimation of the range information. To improve estimation accuracy and satisfy the impact angle constraint, this paper proposes a two-phase optimal guidance law consisting of an observing phase and an attacking phase. In the observing phase, the determinant of Fisher information matrix is maximized to achieve the optimal observability and a suboptimal solution expressed by leading angle is derived analytically. Then, a terminal sliding-mode guidance law is designed to track the desired leading angle. In the followed attacking phase, an optimal guidance law is integrated with a switching term to satisfy both the impact angle constraint and the field-of-view constraint. Finally, comparison studies of the proposed guidance law and a traditional optimal guidance law are conducted on stationary targets and maneuvering targets cases. Simulation results demonstrate that the proposed guidance law is able to improve the range observability and achieve better terminal performances including impact angle accuracy and miss distance.
\end{abstract}

\section{Introduction}

Research on impact angle constrained guidance laws can be divided into two categories, that is, augmented proportional navigation (APN) guidance laws and advanced guidance laws. APN guidance laws can achieve the desired impact angle with only information of line-of-sight (LOS) angle or LOS angular rate by adjusting the proportional navigation coefficient [1] or adding a bias term [2, 3]. However, it is always assumed in APN approaches that the target is stationary or the target's maneuvering path is known. Advanced guidance laws are designed based on modern control theories such as optimal control $[4,5]$, sliding-mode control (SMC) $[6,7]$, and robust control [8]. Compared with APN guidance laws, advanced guidance laws are more flexible to satisfy various requirements.

Nevertheless, the implementation of advanced guidance laws requires the information of range or time to go, which cannot be measured directly by bearings-only sensors. To address the problem, target motion analysis (TMA) is generally employed to estimate range and time to go from noisecorrupted bearings measurements. To enhance estimation accuracy, different filters including extended Kalman filter
(EKF) [9], particle filter (PF) [10, 11], maximum likelihood estimation (MLE), [12] and pseudo-linear estimation [13] were successfully applied in bearings-only TMA. On the other hand, estimation accuracy also relies on state observability which is related to the missile-target relative geometry relation [14-16], so it can be improved via missile trajectory optimization. To improve range observability in a pursuitevasion game problem, the eigenvalue of the error covariance matrix was selected as the optimal criterion and the optimal control command was acquired using enumeration method in [17]. The variances of target-location estimation computed with each missile's measurements were used as the optimal criterion and a suboptimal feedback guidance law was designed using direct-shooting method to enhance the ability of cooperative estimation in [18]. The determinant of Fisher information matrix (FIM) was used as the optimal criterion and the real-time guidance command was generated with vector field theory in [19].

As demonstrated in the aforementioned studies, enhancing system observability can improve estimation accuracy and therefore improve guidance performances. However, it is not trivial to integrate an optimal observability guidance law with an impact angle constrained guidance law, because 


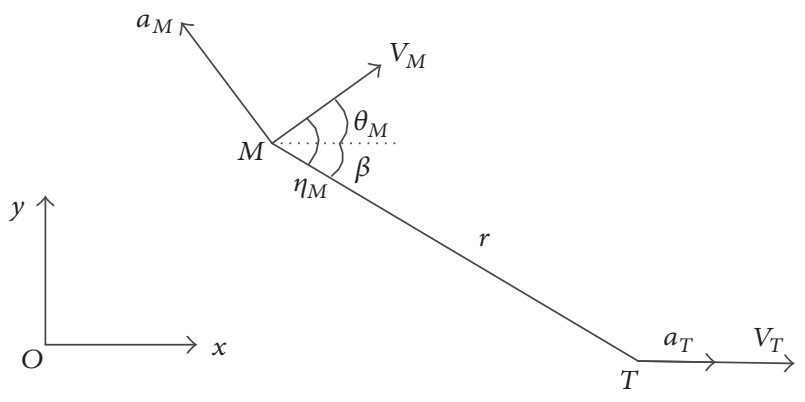

FIgURE 1: Planar missile-target geometry.

the control commands derived by the two guidance laws are generally inconsistent. The LOS angular rate should be maximized to optimize the observability, while zeroing the LOS angular rate is always required in impact angle constrained guidance law. To trade off the requirements of observability enhancement and impact angle constraint, this paper proposes a two-phase optimal guidance law (denoted as TPOGL). The guidance process is divided into an observing phase and an attacking phase. In the observing phase, missile has limited a priori information about the target and the relative distance between missile and target is large, so the missile is guided to maneuver for better observability to decrease estimation errors rapidly. To reduce computation load, the determinant of FIM based on two measurements is chosen as the optimal criterion of the phase. A suboptimal solution expressed by leading angle is derived and tracked by a terminal SMC guidance law. In the attacking phase, the estimation accuracies of states have been largely improved and the relative distance is small, so the missile is guided to maneuver for better striking performances. An optimal guidance law combined with a switching term is employed to satisfy the terminal constraint and the fieldof-view (FOV) constraint. Numerical simulations show that TPOGL can improve the range estimation accuracy and the terminal performances including impact angle accuracy and miss distance. Moreover, the proposed TPOGL has a simple structure and can be implemented in real time.

\section{Problem Statement}

2.1. Engagement Description. The planar missile-target geometry for air-to-ground engagement is depicted in Figure 1, where $r$ is the relative distance between missile and target; $\beta$ is LOS angle; $V_{M}$ and $V_{T}$ are missile and target velocities, respectively; $a_{M}$ and $a_{T}$ are missile and target acceleration, respectively; and $\theta_{M}$ and $\eta_{M}$ are missile flight path angle and leading angle, respectively. Leading angle, LOS angle, and flight path angle satisfy the following relationship:

$$
\eta_{M}=\beta-\theta_{M} .
$$

Denote the missile position as $\mathbf{X}_{M}=\left[x_{M}, y_{M}\right]$ and the target position as $\mathbf{X}_{T}=\left[x_{T}, y_{T}\right]$. Then, LOS angle and missile-target distance can be expressed as

$$
\begin{aligned}
& \beta=\arctan \frac{y_{T}-y_{M}}{x_{T}-x_{M}} \\
& r=\sqrt{\left(x_{T}-x_{M}\right)^{2}+\left(y_{T}-y_{M}\right)^{2} .}
\end{aligned}
$$

It is assumed that missile speed is constant and the target is moving along $x$-axis. Thus, we can get $a_{M}=V_{M} \dot{\theta}_{M}, \dot{\theta}_{T}=0$, and $a_{T}=\dot{V}_{T}$. Then the planar engagement is described by the following differential equations:

$$
\begin{aligned}
\dot{r} & =-V_{M} \cos \left(\beta-\theta_{M}\right)+V_{T} \cos \left(\beta-\theta_{T}\right) \\
\dot{\beta} & =\frac{1}{r}\left(V_{M} \sin \left(\beta-\theta_{M}\right)-V_{T} \sin \left(\beta-\theta_{T}\right)\right) \\
\dot{\theta}_{M} & =\frac{a_{M}}{V_{M}} \\
\dot{V}_{T} & =a_{T} .
\end{aligned}
$$

To maintain the target within seeker's FOV, missile leading angle cannot exceed seeker's FOV $\psi$, which is expressed as

$$
\left|\eta_{M}\right| \leq \psi
$$

2.2. Estimation Model. In modified polar (MP) coordinates [9], the dynamic equations of the bearings-only localization problem are formulated as

$$
\begin{aligned}
& {\left[\begin{array}{c}
\dot{\beta} \\
\dot{r} \\
\frac{r}{\beta} \\
\frac{1}{r}
\end{array}\right]^{\prime} } {\left[\begin{array}{c}
\frac{-2 \dot{\beta} \dot{r}}{r}-\frac{\cos \left(\beta-\theta_{M}\right)}{r} a_{M} \\
\dot{\beta}^{2}-\left(\frac{\dot{r}}{r}\right)^{2}-\frac{\sin ^{2}\left(\beta-\theta_{M}\right)}{r} a_{M} \\
\dot{\beta} \\
-\frac{\dot{r}}{r^{2}}
\end{array}\right] } \\
&+\frac{1}{r}\left[\begin{array}{c}
-\sin \left(\beta-\theta_{T}\right) \\
\cos \left(\beta-\theta_{T}\right) \\
0 \\
0
\end{array}\right] a_{T} .
\end{aligned}
$$

During the guidance process, missile state $\theta_{M}$ is assumed to be accurately measured by onboard devices, while missiletarget relative states including $\beta$ and $r$ need to be estimated from noise-corrupted measurements. Missile acceleration $a_{M}$ is the control variable of the system and generated by the guidance system. Target acceleration $a_{T}$ is treated as noise to the system.

Then the state equations for the state estimation problem are

$$
\dot{\mathbf{x}}=\left[\begin{array}{c}
-2 x_{1} x_{2}-x_{4} \cos \left(x_{3}-\theta_{M}\right) u \\
x_{1}^{2}-x_{2}^{2}-x_{4} \sin \left(x_{3}-\theta_{M}\right) u \\
x_{1} \\
-x_{2} x_{4}
\end{array}\right]+\boldsymbol{\omega},
$$


where $\mathbf{x}=\left[x_{1}, x_{2}, x_{3}, x_{4}\right]^{\mathrm{T}}=[\dot{\beta}, \dot{r} / r, \beta, 1 / r]^{\mathrm{T}}$ is the state vector, $u$ is the control variable (i.e., missile acceleration), and $\boldsymbol{\omega}$ is the process noise related to target maneuvering. lem is

The measurement equation for the state estimation prob-

$$
z=x_{3}+v,
$$

where $z$ is the measurement of LOS angle and $v$ is assumed to be a zero-mean white Gaussian noise with variance of $\sigma^{2}$.

\section{Two-Phase Optimal Guidance Law Design}

In this section, we propose a two-phase optimal guidance law to make tradeoff between impact angle constrained guidance law and optimal observability guidance law. And FOV constraint is considered in both phases to obtain a practical guidance law.

3.1. Optimal Observability Guidance Law Design for the Observing Phase. To maximize the range observability for the missile-target engagement, the determinant of FIM is chosen as the optimal criterion [20] and the suboptimal leading angle is derived analytically. And a guidance law based on terminal SMC theory is designed to keep the missile flying with the desired leading angle.

3.1.1. Optimal Criterion. FIM describes the amount of information that the state vector carries about the measurement. And FIM can be expressed as [20]

$$
\mathrm{FIM}=-E\left[\frac{\partial^{2}}{\partial \chi} \ln p(\Theta \mid \chi)\right],
$$

where $p(\Theta \mid \chi)$ denotes the probability density function of the measurement $\Theta$ given the states $\chi$. For the bearingsonly localization problem, the missile-target relative position $\Delta \mathbf{X}=\mathbf{X}_{T}-\mathbf{X}_{M}=\left[x_{T}-x_{M}, y_{T}-y_{M}\right]$ and the LOS angle $\beta$ are taken as states and measurement, respectively. As described in (7), the measurement noise is assumed to be a zero-mean Gaussian white noise with constant variance $\sigma^{2}$; then the probability density function $p(\beta \mid \Delta \mathbf{X})$ in this work is

$$
\begin{aligned}
& p(\beta \mid \Delta \mathbf{X}) \\
& \quad=\frac{1}{\sqrt{2 \pi} \sigma} \exp \left[\frac{-(\beta-h(\Delta \mathbf{X}))(\beta-h(\Delta \mathbf{X}))^{\mathrm{T}}}{2 \sigma^{2}}\right],
\end{aligned}
$$

where $h(\Delta \mathbf{X})=\arctan \left[\left(y_{T}-y_{M}\right) /\left(x_{T}-x_{M}\right)\right]$.

Substituting (2) and (9) into (8), FIM for the bearingsonly localization problem can be simplified as [21]

$$
\begin{aligned}
\text { FIM } & =\frac{1}{\sigma^{2}} \int_{0}^{T}\left(\frac{\partial h(\Delta \mathbf{X})}{\partial \Delta \mathbf{X}}\right)\left(\frac{\partial h(\Delta \mathbf{X})}{\partial \Delta \mathbf{X}}\right)^{T} d \tau \\
& =\frac{1}{\sigma^{2}}\left[\begin{array}{cc}
\int_{0}^{T} \frac{\sin ^{2} \beta}{r^{2}} d \tau & -\frac{1}{2} \int_{0}^{T} \frac{\sin 2 \beta}{r^{2}} d \tau \\
-\frac{1}{2} \int_{0}^{T} \frac{\sin 2 \beta}{r^{2}} d \tau & \int_{0}^{T} \frac{\cos ^{2} \beta}{r^{2}} d \tau
\end{array}\right],
\end{aligned}
$$

where $T$ is the total time of the guidance process.
From (10), computation of FIM is complex due to the integral process. To reduce computing load, limited number of measurements can be used to obtain a suboptimal solution of FIM $[19,22]$ and this paper uses two-step measurements.

Assume the step length $h$ between two measurements is small; then missile leading angle and target position could be considered as constant during a single step [21]. Integrating (3) during time $h$, we can get

$$
\begin{aligned}
& r_{2}=r_{1}-V_{M} \cos \eta_{M} h \\
& \beta_{2}=\beta_{1}+\tan \eta_{M} \ln \left(\frac{r_{1}}{r_{1}-V_{M} \cos \eta_{M} h}\right),
\end{aligned}
$$

where $r_{1}$ and $\beta_{1}$ are the range and LOS angle at $t_{k}$ and $r_{2}$ and $\beta_{2}$ are the range and LOS angle at $t_{k+1}$.

Then, each term of FIM in (7) can be integrated as follows:

$$
\begin{aligned}
& \int_{0}^{h} \frac{\sin ^{2} \beta}{r^{2}} d t=-\frac{1}{2 V_{M} \cos \eta_{M}}\left(\frac{1}{r_{2}}-\frac{1}{r_{1}}\right) \\
& -\frac{1}{V_{M}\left(4 \sin ^{2} \eta_{M}+\cos ^{2} \eta_{M}\right)}\left[\sin \eta_{M}\right. \\
& \cdot\left(\frac{\sin 2 \beta_{2}}{r_{2}}-\frac{\sin 2 \beta_{1}}{r_{1}}\right)+ \\
& \left.\cdot \frac{\cos \eta_{M}}{2}\left(\frac{\cos 2 \beta_{2}}{r_{2}}-\frac{\cos 2 \beta_{1}}{r_{1}}\right)\right] \\
& \int_{0}^{h} \frac{\cos \beta^{2}}{r^{2}} d t=-\frac{1}{2 V_{M} \cos \eta_{M}}\left(\frac{1}{r_{2}}-\frac{1}{r_{1}}\right) \\
& +\frac{1}{V_{M}\left(4 \sin ^{2} \eta_{M}+\cos ^{2} \eta_{M}\right)}\left[\sin \eta_{M}\right. \\
& \cdot\left(\frac{\sin 2 \beta_{2}}{r_{2}}-\frac{\sin 2 \beta_{1}}{r_{1}}\right) \\
& \left.+\frac{\cos \eta_{M}}{2}\left(\frac{\cos 2 \beta_{2}}{r_{2}}-\frac{\cos 2 \beta_{1}}{r_{1}}\right)\right] \\
& \int_{0}^{h} \frac{\sin 2 \beta}{r^{2}} d t=\frac{1}{V_{M}\left(4 \sin ^{2} \eta_{M}+\cos ^{2} \eta_{M}\right)}\left[-2 \sin \eta_{M}\right. \\
& \cdot\left(\frac{\cos 2 \beta_{2}}{r_{2}}-\frac{\cos 2 \beta_{1}}{r_{1}}\right)+\cos \eta_{M} \\
& \left.\quad\left(\frac{\sin 2 \beta_{2}}{r_{2}}-\frac{\sin 2 \beta_{1}}{r_{1}}\right)\right] \cdot
\end{aligned}
$$

Then, the determinant of FIM can be expressed as

|FIM|

$$
\begin{aligned}
& =\frac{4}{\sigma^{2} V_{M}{ }^{2} r_{1}^{2}\left(3 \sin ^{2} \eta_{M}+1\right) \cos ^{2} \eta_{M}}\left[\left(\frac{r_{1}-r_{2}}{r_{2}}\right)^{2}\right. \\
& \left.\cdot \sin ^{2} \eta_{M}+\frac{r_{1}}{r_{2}} \cos ^{2} \eta_{M} \sin ^{2}\left(\ln \left(\frac{r_{1}}{r_{2}}\right) \tan \eta_{M}\right)\right] .
\end{aligned}
$$




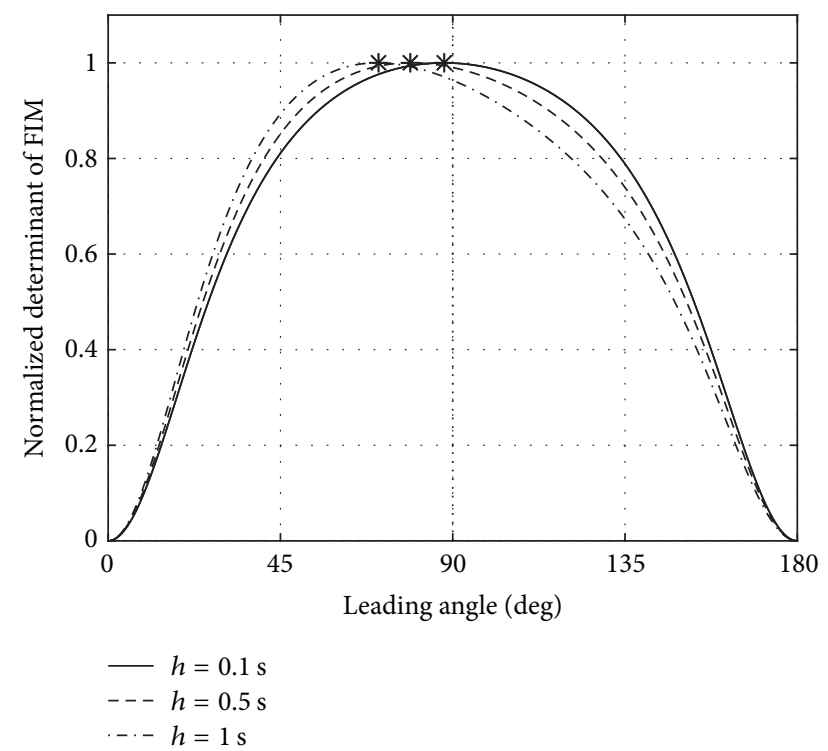

FIGURE 2: Normalized determinant of FIM with respect to leading angle.

According to (11) and (13), when states at $t_{k}$ are fixed, determinant of FIM is only related to leading angle and step length, and it is an even function of leading angle. To illustrate the relationship between determinant of FIM and leading angle, define the normalized determinant of FIM as

$$
\left|\operatorname{FIM}\left(\eta_{M}\right)\right|^{*}=\frac{\left|\operatorname{FIM}\left(\eta_{M}\right)\right|}{\max \left(|\operatorname{FIM}|\left(\eta_{M}\right)\right)}
$$

Figure 2 shows the normalized determinant of FIM varying with leading angle when $r_{1}=2000 \mathrm{~m}$ and $V_{M}=200 \mathrm{~m} / \mathrm{s}$. From Figure 2, for a specified step length, there is an optimal leading angle within $\left(0^{\circ}, 90^{\circ}\right)$ which maximizes the normalized determinant of FIM. Using the line search method, the optimal leading angle with respect to the step length can be obtained, as shown in Figure 3.

From Figure 3, the optimal leading angle approaches $90^{\circ}$ when the step length approaches zero. For a bearings-only seeker of a missile, the update time (i.e., step length) is usually less than $0.01 \mathrm{~s}$, so the optimal leading angle is near $90^{\circ}$. The optimal leading angle is also limited by seeker's FOV which is far less than $90^{\circ}$. Thus, the optimal leading angle should coincide with seeker's FOV to maximize the trajectory observability. Furthermore, considering the estimation errors and target maneuvering, there should be a margin between the optimal leading angle and seeker's FOV to ensure the missile lock-on. The margin is determined according to seeker performances and target maneuvering.

3.1.2. Guidance Law Design Using Terminal SMC. To drive leading angle to its desired value, a guidance law with finite time convergence is designed based on terminal SMC theory. The switching surface is chosen as the tracking error of leading angle

$$
s=\widetilde{\eta}_{M},
$$

where $\tilde{\eta}_{M}=\eta_{M}-\eta_{M}^{*}$ and $\eta_{M}^{*}$ is the optimal leading angle.

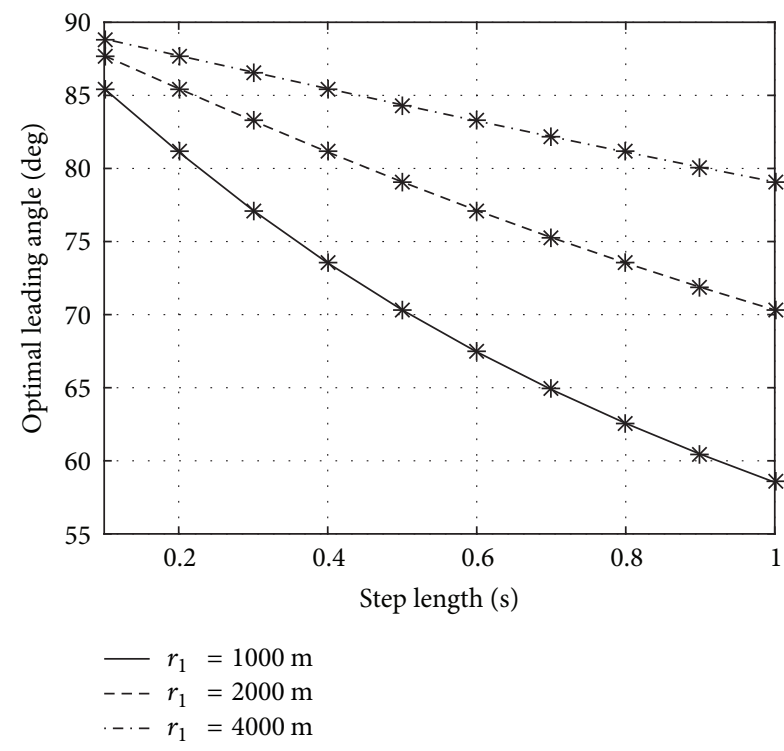

Figure 3: Optimal leading angle with respect to step length.

The reaching law is selected as

$$
\dot{s}=-k \operatorname{sgn}(s)|s|^{\alpha}, \quad k>0,0<\alpha<1 .
$$

Substituting (1) and (3) into (16) yields

$$
a_{M}=V_{M}\left(\dot{\beta}+k \operatorname{sgn}(s)|s|^{\alpha}\right) .
$$

Replacing the target-related states with the estimated states, the actual guidance command in the observing phase is

$$
u_{\text {observe }}=V_{M}\left(x_{1}+k \operatorname{sgn}(s)|s|^{\alpha}\right),
$$

where $s=x_{3}-\theta_{M}-\eta_{M}^{*}$.

To prove the finite time convergence of the proposed guidance law, consider a Lyapunov function as

$$
V=\frac{s^{2}}{2} \text {. }
$$

Differentiating (19), the result is

$$
\dot{V}=s \dot{s}=s\left(\dot{\beta}-\dot{\theta}_{M}\right)
$$

where $\dot{\beta}$ is the accurate LOS angular rate.

Considering estimation errors of the states and substituting (3) into (20), the result is

$$
\dot{V}=s\left(x_{1}+e_{x 1}-\frac{u_{\text {observe }}}{V_{M}}\right),
$$

where $x_{1}$ and $e_{x 1}$ denote the estimated LOS angular rate and the estimation error of LOS angular rate, respectively.

Substituting (18) into (21), the result is

$$
\dot{V}=s\left(e_{x 1}-k \operatorname{sgn}(s)|s|^{\alpha}\right) .
$$


(A) If $e_{x 1}$ is zero, (22) can be expressed in the following form:

$$
\dot{V}=-k \operatorname{sgn}(s)|s|^{\alpha} s .
$$

Substituting (19) into (23), the result is

$$
\dot{V}=-2^{(\alpha+1) / 2} k V^{(\alpha+1) / 2} \text {. }
$$

From (16), we can get $0.5<(\alpha+1) / 2<1$ and $-2^{(\alpha+1) / 2} k<0$. According to finite time convergence theory, the system can converge to its equilibrium point in a finite time and the convergence time is

$$
T_{\mathrm{c}}=\frac{V^{(1-\alpha) / 2}}{2^{(\alpha-1) / 2} k} \text {. }
$$

(B) If $e_{x 1}$ is not zero, (22) can be expressed in the following form:

$$
\dot{V}=s\left(\left(\frac{e_{x 1}}{\operatorname{sgn}(s)|s|^{\alpha}}-k\right) \operatorname{sgn}(s)|s|^{\alpha}\right) .
$$

If the parameters of guidance law are designed to satisfy $e_{x 1} / \operatorname{sgn}(s)|s|^{\alpha}<k$, (26) has a similar structure to (24). Then the system can converge to the neighborhood of the switching surface which is

$$
|s| \leq\left(\frac{e_{x 1}}{k}\right)^{1 / \alpha}
$$

To sum up, if the estimation error of LOS angular rate is zero, the leading angle tracking error will converge to zero in finite time; if the estimation error of LOS angular rate is not zero and the parameters are well designed, the leading angle tracking error can converge to the neighborhood of zero within finite time.

According to (18), computation of the guidance command in the observing phase only needs the estimated states $x_{1}$ and $x_{3}$, which are LOS-angle-related states, and it is independent of $x_{2}$ and $x_{4}$, which are range-related states. Because of the linearity between LOS-angle-related states and measurement of bearings-only seekers, estimation errors of the LOS-angle-related states could converge rapidly while the estimation errors of range-related states cannot. In the observing phase, using only the LOS-angle-related states as inputs of the guidance command makes the guidance law insensitive to the initial guesses and improves the robustness of the guidance law.

3.2. Optimal Guidance Law Design for the Attacking Phase. In the attacking phase, the missile is guided to maneuver to attack the target with desired performances. The optimal guidance law in [4] is used to satisfy the impact angle constraint and it is expressed as

$$
a_{M}=\frac{V_{M}}{t_{\mathrm{go}}}\left(-N_{1} \beta+N_{2} \theta_{M}+N_{3} \theta_{M f}\right),
$$

where $t_{\text {go }}$ is time to go, $\theta_{M f}$ is the desired impact angle, and

$$
\begin{aligned}
& N_{1}=(N+2)(N+3) \\
& N_{2}=2(N+2) \\
& N_{3}=(N+1)(N+2),
\end{aligned}
$$

$$
N>0 .
$$

Simply replacing $t_{\text {go }}$ with $r / \dot{r}$, the guidance law is rewritten as

$$
a_{M}=V_{M} \frac{\dot{r}}{r}\left(-N_{1} \beta+N_{2} \theta_{M}+N_{3} \theta_{M f}\right) .
$$

Replacing the target-related states with the estimated states denoted in (5), the actual guidance command in the attacking phase is

$$
u_{\text {attack }}=V_{M} x_{2}\left(-N_{1} x_{3}+N_{2} \theta_{M}+N_{3} \theta_{M f}\right) .
$$

Because FOV constraint is not considered in the above optimal guidance law, a switching term is added to prevent the leading angle from exceeding seeker's FOV. The switching term works when the leading angle approaches seeker's FOV and it is designed as

$$
a_{M}=V_{M}\left(\dot{\beta}+\operatorname{sgn}\left(\eta_{M}\right) c_{1}\right), \quad c_{1}>0
$$

Replacing the target-related states with the estimated states, the actual guidance command for switching term is

$$
u_{\text {switch }}=V_{M}\left(x_{1}+\operatorname{sgn}\left(x_{3}-\theta_{M}\right) c_{1}\right) .
$$

To prove the effectiveness of the switching term, consider a Lyapunov function as

$$
V=\frac{\eta_{M}^{2}}{2}
$$

Differentiating (34) yields

$$
\dot{V}=\eta_{M} \dot{\eta}_{M}=\left(\beta-\theta_{M}\right)\left(\dot{\beta}-\dot{\theta}_{M}\right) \text {. }
$$

Considering the estimation errors of the states and substituting (1), (3), and (33) into (35), the result is

$$
\begin{aligned}
\dot{V} & =\left(x_{3}+e_{x 3}-\theta_{M}\right)\left(x_{1}+e_{x 1}-\frac{u_{\text {switch }}}{V_{M}}\right) \\
& =\left(x_{3}-\theta_{M}+e_{x 3}\right)\left(e_{x 1}-\operatorname{sgn}\left(x_{3}-\theta_{M}\right) c_{1}\right) \\
& =\left(\widehat{\eta}_{M}+e_{x 3}\right)\left(e_{x 1}-\operatorname{sgn}\left(\widehat{\eta}_{M}\right) c_{1}\right),
\end{aligned}
$$

where $\widehat{\eta}_{M}$ is the estimated leading angle and $e_{x 3}$ and $e_{x 1}$ are the estimation errors of LOS angle and LOS angular rate, respectively. Since the switching term only works when the leading angle is close to seeker's FOV, $e_{x 3}$ is negligible compared to $\widehat{\eta}_{M}$. Thus, (36) can be rewritten as

$$
\dot{V}=\widehat{\eta}_{M}\left(e_{x 1}-\operatorname{sgn}\left(\widehat{\eta}_{M}\right) c_{1}\right)
$$


According to (37), when $c_{1}>\left|e_{x 1}\right|$, we can get $\dot{V}<0$, so the value of the leading angle will decrease. Thus, if leading angle gets very close to seeker's FOV, the leading angle will decrease and cannot exceed seeker's FOV by activating the switching term. Besides, because leading angle will gradually decrease to zero under the impact angle constrained guidance law, the switching term will not work when the range is small and will not affect the terminal performances.

To sum up, the employed guidance command in the attacking phase is expressed as

$$
u= \begin{cases}u_{\text {attack }}, & || \eta_{M}|-\psi| \leq c_{2} \\ u_{\text {switch }}, & || \eta_{M}|-\psi|>c_{2}\end{cases}
$$

For convenience, a unified form of the attacking guidance law is given as

$$
u=\varepsilon(\Delta \eta) u_{\mathrm{attack}}+\varepsilon(-\Delta \eta) u_{\mathrm{switch}}
$$

where $\varepsilon(\cdot)$ is the step function and $\Delta \eta$ is the indicator of whether the switching guidance law should be used in the attacking phase, and it is expressed as

$$
\Delta \eta=|| \eta_{M}|-\psi|-c_{2}, \quad c_{2}>0
$$

3.3. Summary. Now, the guidance laws for the observing phase and the attacking phase are designed, respectively. The handover condition of the two phases is related to missiletarget relative states, impact angle, dynamic constraint, and FOV constraint. In this work, the missile-target relative distance is used as the handover condition for simplicity. Then, TPOGL is summarized as follows:

$$
a_{M}= \begin{cases}V_{M}\left(\dot{\beta}+k \operatorname{sgn}\left(\widetilde{\eta}_{M}\right)\left|\widetilde{\eta}_{M}\right|^{\alpha}\right), & r>r_{h} \\ V_{M}\left(\varepsilon(\Delta \eta) \frac{\dot{r}}{r}\left(-N_{1} \beta+N_{2} \theta_{M}+N_{3} \theta_{M f}\right)+\varepsilon(-\Delta \eta)\left(\dot{\beta}+\operatorname{sgn}\left(\eta_{M}\right) c_{1}\right)\right), & r \leq r_{h},\end{cases}
$$

where $r_{h}$ is the threshold of the missile-target distance for guidance laws handover and it is determined via trial and error.
The actual guidance command using estimated information is

$$
u= \begin{cases}V_{M}\left(x_{1}+k \operatorname{sgn}\left(x_{3}-\theta_{M}-\eta_{M}^{*}\right)\left|x_{3}-\theta_{M}-\eta_{M}^{*}\right|^{\alpha}\right), & x_{4}<\frac{1}{r_{h}} \\ V_{M}\left(\varepsilon(\Delta \eta) x_{2}\left(-N_{1} x_{3}+N_{2} \theta_{M}+N_{3} \theta_{M f}\right)+\varepsilon(-\Delta \eta)\left(x_{1}+\operatorname{sgn}\left(x_{3}-\theta_{M}\right) c_{1}\right)\right), & x_{4} \geq \frac{1}{r_{h}} .\end{cases}
$$

\section{Simulation}

In this section, the simulation results are provided to demonstrate the effectiveness of TPOGL. To show the appealing performances of TPOGL, it is tested under different impact angle constraints and then compared with the optimal guidance law (OGL) described in (39).

4.1. Engagement Scenarios. In all the scenarios, the engagements start with the missile at $(0,1000 \mathrm{~m})$ and the target at $(3000 \mathrm{~m}, 0)$. The missile's initial path angle is $0^{\circ}$ and it flies at a constant speed of $200 \mathrm{~m} / \mathrm{s}$ with a maximum acceleration of $70 \mathrm{~m} / \mathrm{s}^{2}$. The seeker's FOV is $30^{\circ}$, and the optimal leading angle is set as $29^{\circ}$ considering the disturbances in the guidance.

For each simulation of both guidance laws, the states in (6) are estimated using EKF and initialized as (43) without knowledge of the target since TPOGL is not sensitive to initial guesses.

$$
\widehat{\mathbf{x}}_{0 \mid 0}=\left[0, \frac{V_{M}}{r_{\max }}, \beta(0), \frac{1}{r_{\max }}\right]^{\mathrm{T}},
$$

where $r_{\max }$ is seeker's maximum detection range and $\beta(0)$ is the initial measurement of LOS angle. Guidance parameters used in the guidance laws are given in Table 1.

4.2. Simulations under Different Impact Angle Constraints. In this subsection, simulations are conducted to test the performances of TPOGL under different impact angle constraints, that is, $-30^{\circ},-60^{\circ}$, and $-90^{\circ}$. The handover distances for different impact angle constraints are given in Table 2.

The simulation results of the trajectories, states, and guidance commands are shown in Figure 4. From Figure 4, in the observing phase, the states and guidance commands of different cases are basically the same. That is because the guidance command of the observing phase is only related to the leading angle and LOS angle, and it is independent of desired impact angle. When the estimated missile-target relative distance reached the predefined handover distance, the missile turns to the attacking phase and then maneuvers to attack the target with desired impact angle. The simulation results show that TPOGL is able to strike the target with desired impact angles under dynamics and FOV constraints. 

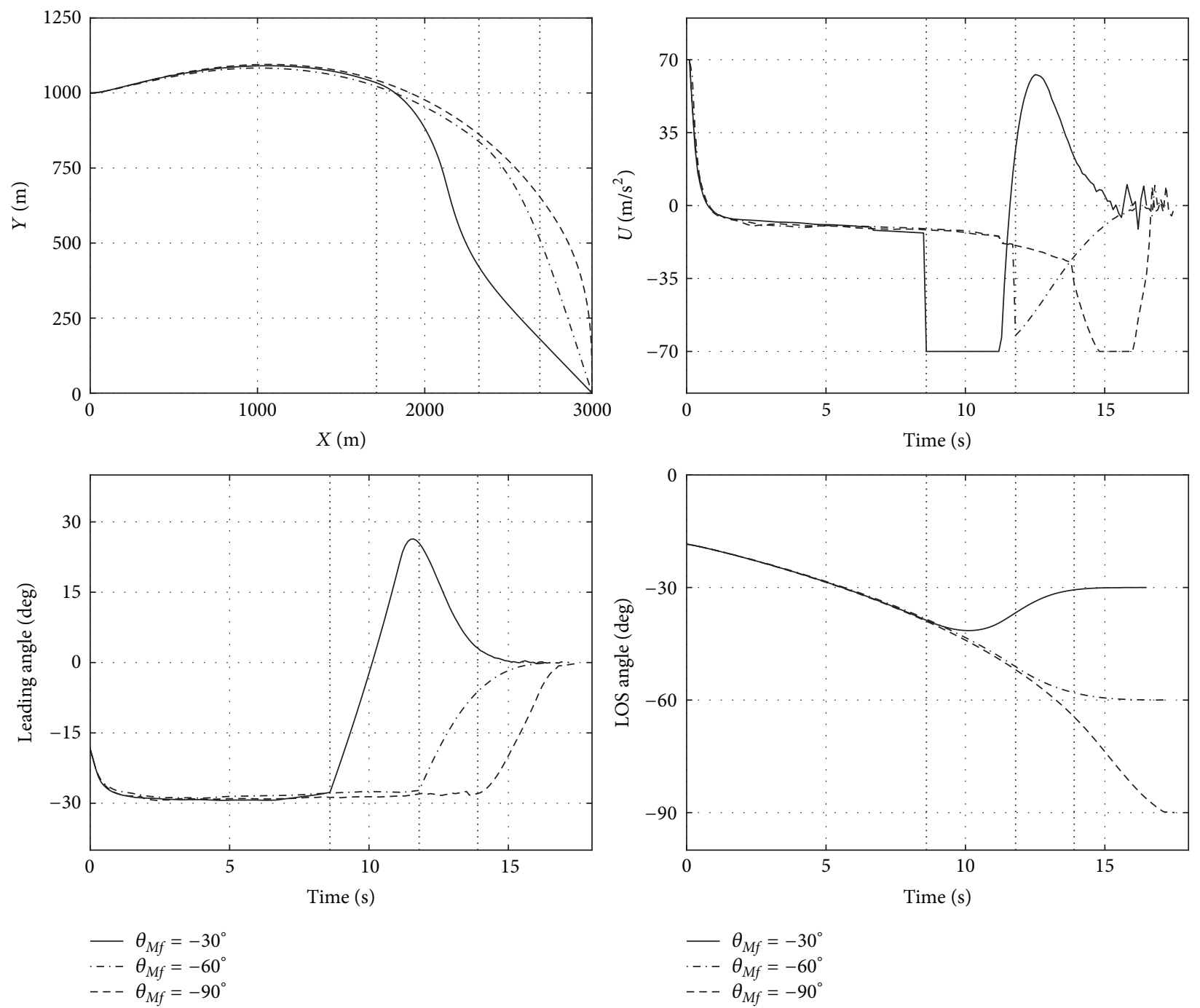

FIGURE 4: Simulation results comparisons under different impact angle constraints.

TABLE 1: Guidance parameters.

\begin{tabular}{lccc}
\hline Parameter & Value & Parameter & Value \\
\hline$k$ & 2 & $\alpha$ & 0.6 \\
$c_{1}$ & 0.01 & $c_{2}$ & $0.2^{\circ}$ \\
$N$ & 5 & $\sigma$ & $1^{\circ}$ \\
$r_{\max }$ & $4000 \mathrm{~m}$ & & \\
\hline
\end{tabular}

Monte Carlo samples are carried out to demonstrate the statistical characteristics of TPOGL under different impact angle constraints. Figure 5 shows the distributions of miss distances and impact angle errors from 100 Monte Carlo samples and each sample differs from the others with random measurement noises.

From Figure 5, the median values of miss distances of the three cases are $0.12 \mathrm{~m}, 0.11 \mathrm{~m}$, and $0.15 \mathrm{~m}$, respectively. The median values of impact angle errors of the three cases are $0.41^{\circ}, 0.37^{\circ}$, and $0.52^{\circ}$, respectively. The distributions of the miss distances are mostly within $(0,0.5 \mathrm{~m})$ and the
TABLE 2: Impact angles and handover distances.

\begin{tabular}{lcc}
\hline Case & Impact angle & Handover distance \\
\hline 1 & $-30^{\circ}$ & $2000 \mathrm{~m}$ \\
2 & $-60^{\circ}$ & $1200 \mathrm{~m}$ \\
3 & $-90^{\circ}$ & $700 \mathrm{~m}$ \\
\hline
\end{tabular}

distributions of impact angle errors are mostly within $\left(0,1^{\circ}\right)$. The results of different impact angle cases show that TPOGL can strike the target with desired impact angle accurately.

4.3. Performance Comparisons of TPOGL and OGL. In this subsection, TPOGL is compared with OGL against stationary targets and maneuvering targets, respectively. The desired impact angle is set as $-30^{\circ}$ and the handover distance for TPOGL is set as $2000 \mathrm{~m}$.

4.3.1. Stationary Targets Interception Case. The states and actual guidance commands of different guidance laws are demonstrated in Figure 6. The estimation errors of range $r$, 

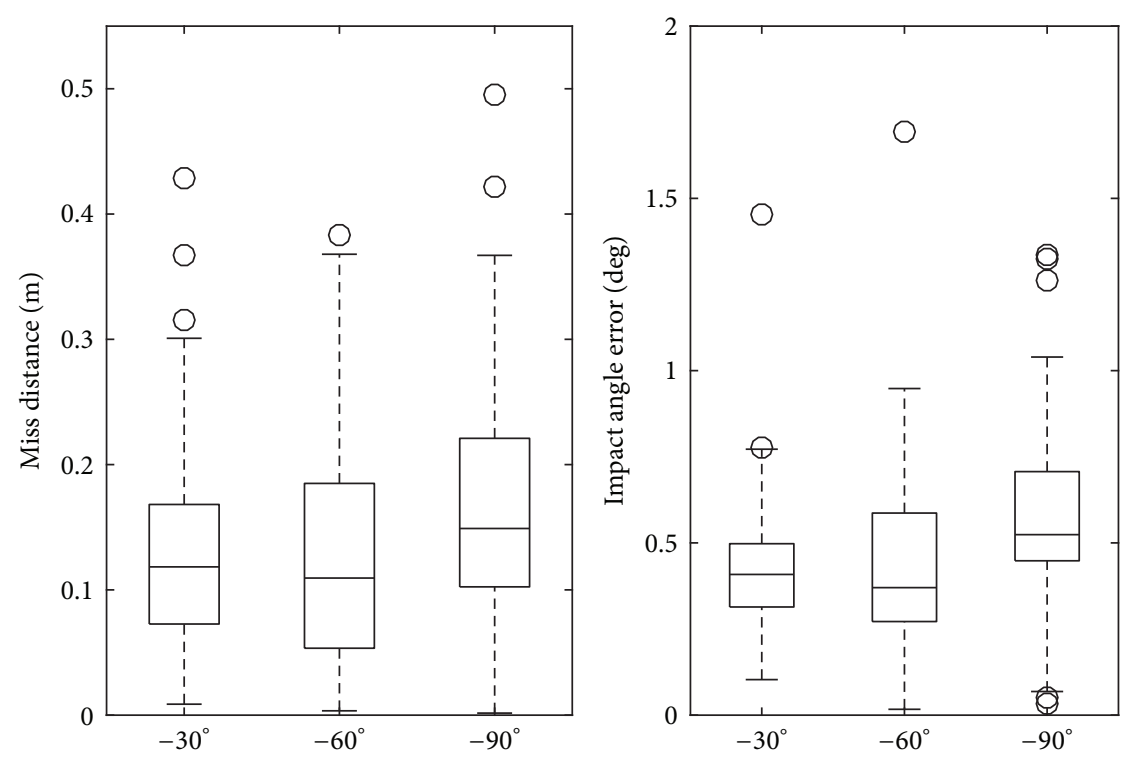

FIGURE 5: Distributions of errors under different impact angle constraints.
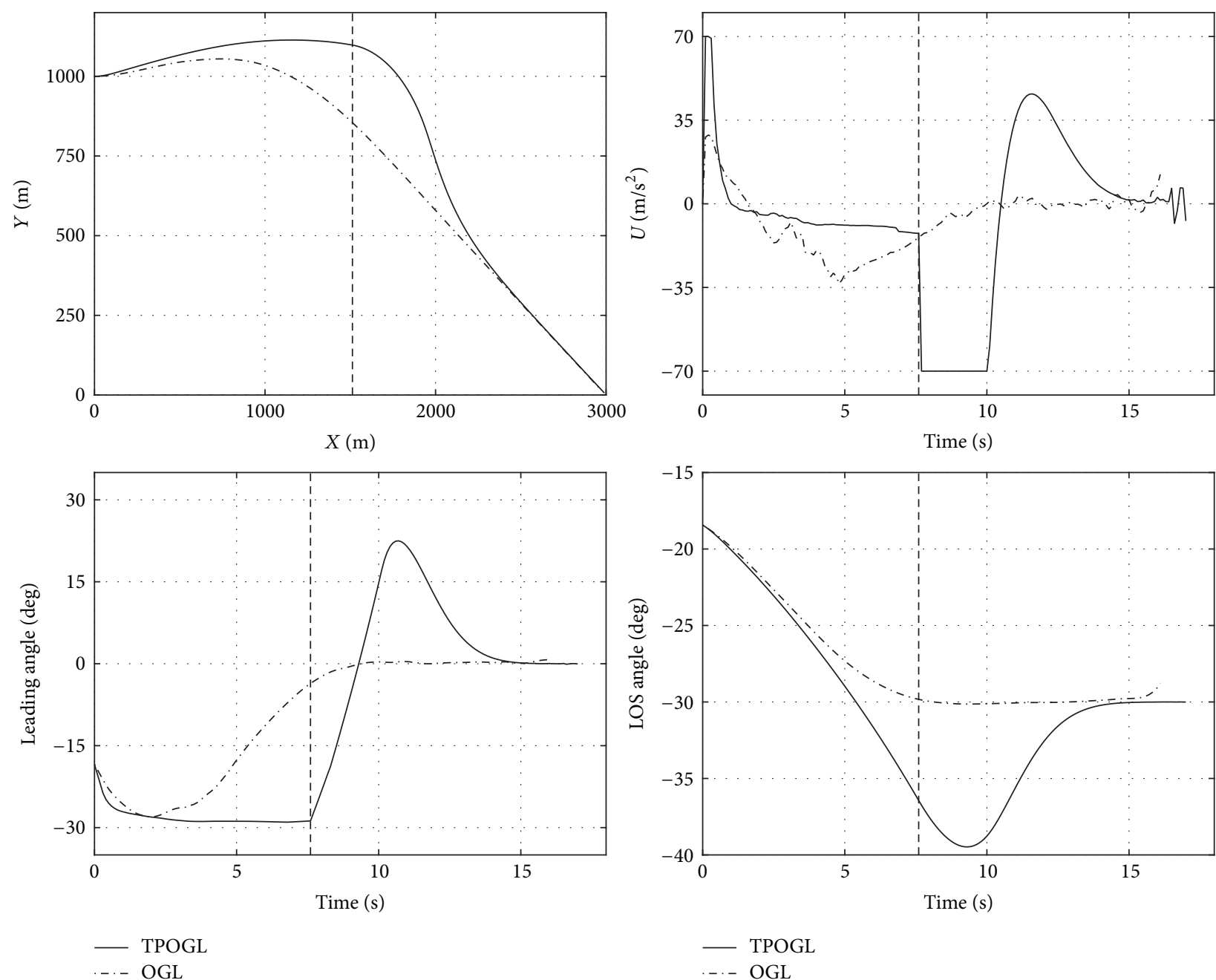

FIGURE 6: Simulation results comparisons against stationary targets. 

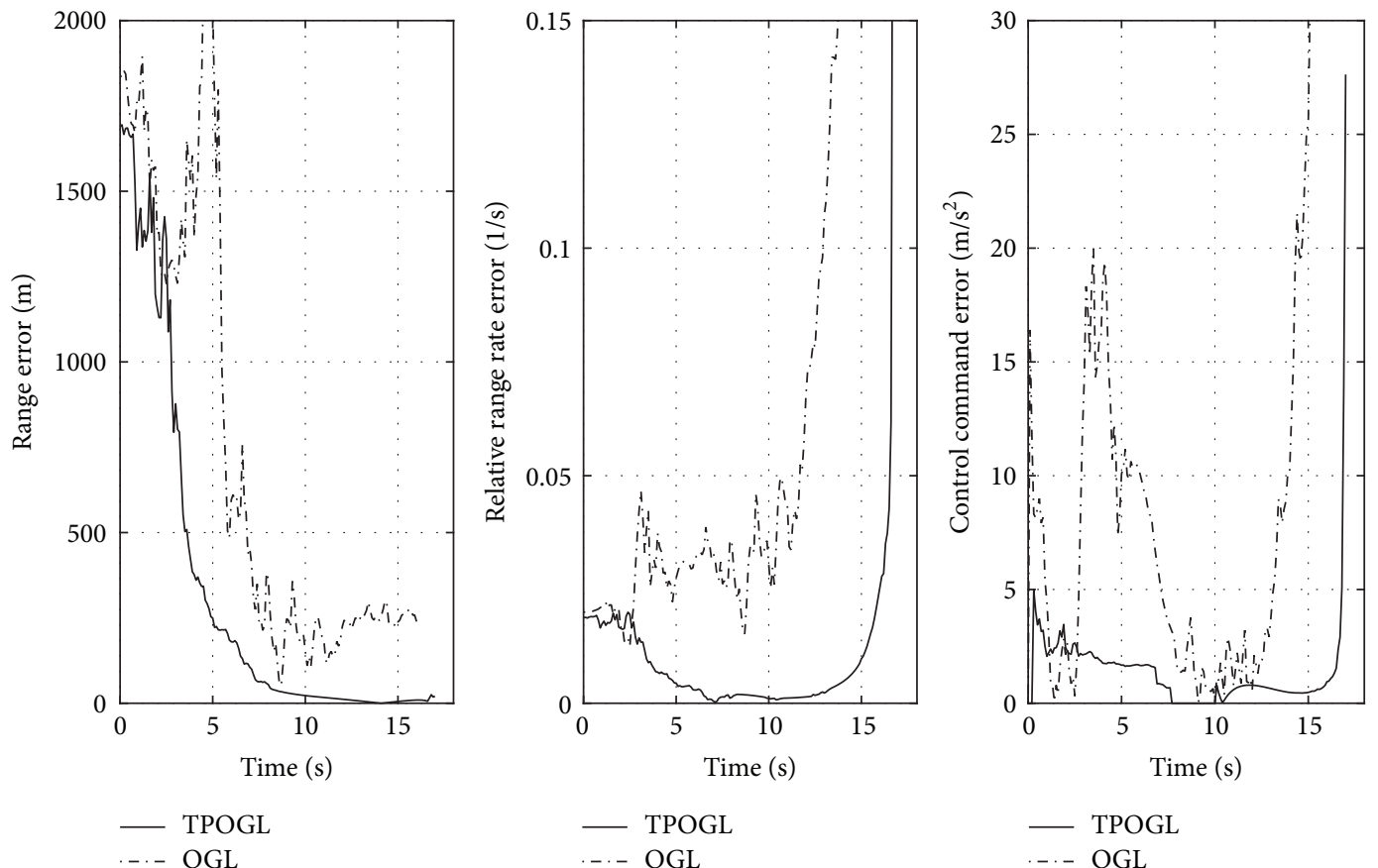

FIGURE 7: Estimation errors comparisons against stationary targets.

relative range rate $\dot{r} / r$, and guidance command errors are shown in Figure 7.

From Figure 6, compared with OGL, trajectory of TPOGL needs a larger maneuver due to observability requirement. Missile with TPOGL turns from observing phase to attacking phase when the distance decreases to $2000 \mathrm{~m}$ at $7.6 \mathrm{~s}$ as the dash line shows in the figure. In the observing phase, the LOS angle obtained by TPOGL varies rapidly as the leading angle reaches its bound and keeps close to it. In the attacking phase, the leading angle gradually varies to zero and the LOS angle is driven to its desired value. At the end of the guidance, the LOS angle obtained by TPOGL is very close to the desired impact angle, while that obtained by OGL departures from $-30^{\circ}$ because of the large guidance command error.

From Figure 7, the range error obtained by TPOGL decreases rapidly to zero, while the range error obtained by OGL does not converge and keeps high. The relative range rate error obtained by TPOGL is small most of the time, but it diverges at the end of the guidance because the accurate value of the relative range rate approaches infinity. The relative range rate error obtained by OGL cannot converge and diverges to infinity much earlier than TPOGL. The guidance command error shows the difference between the actual guidance command which is computed using estimated values of states and the ideal guidance command which is computed using accurate value of states. And the guidance command error obtained by TPOGL is small at the very beginning of the guidance because of the rapid convergence of the LOS-angle-related states. The actual guidance command obtained by TPOGL keeps close to the ideal guidance command, guiding the missile to hit target with desired performances.
Monte Carlo samples are carried out to demonstrate the statistical characteristics of the guidance laws. Comparisons are made on a set of 100 Monte Carlo samples and each sample differs from the others with random measurement noises. The boxplots of miss distances and impact angle errors against stationary targets are depicted in Figure 8.

From Figure 8, the median value of miss distances obtained by TPOGL is $0.12 \mathrm{~m}$ while that obtained by OGL is $0.21 \mathrm{~m}$, and TPOGL improves the accuracy by $45 \%$. The median value of impact angle errors obtained by TPOGL is $0.34^{\circ}$ while that obtained by OGL is $1.31^{\circ}$, and TPOGL improves the accuracy by $75 \%$. The distributions of the errors obtained by TPOGL are more concentrated compared to those obtained by OGL, indicating that TPOGL has better robustness.

4.3.2. Maneuvering Targets Interception Case. In this case, the target has an initial velocity of $10 \mathrm{~m} / \mathrm{s}$ along $x$-axis and its acceleration is a process noise belonging to a uniform distribution within $[-10,10]$.

The simulation results of TPOGL and OGL against maneuvering targets are shown in Figure 9. Estimation errors and guidance command errors are given in Figure 10.

From Figure 9, TPOGL also can obtain the desired performances for maneuvering targets interception. The value of leading angle never exceeds $30^{\circ}$, indicating that the target is kept in seeker's FOV. Leading angle converges to zero and LOS angle converges to $-30^{\circ}$, which means the impact angle constraint is fulfilled. But for OGL, leading angle and LOS angle both have distinct errors with their desired values.

From Figure 10, the errors obtained by TPOGL are close to those in the stationary case, which means that states can be estimated accurately with random target maneuvering. 

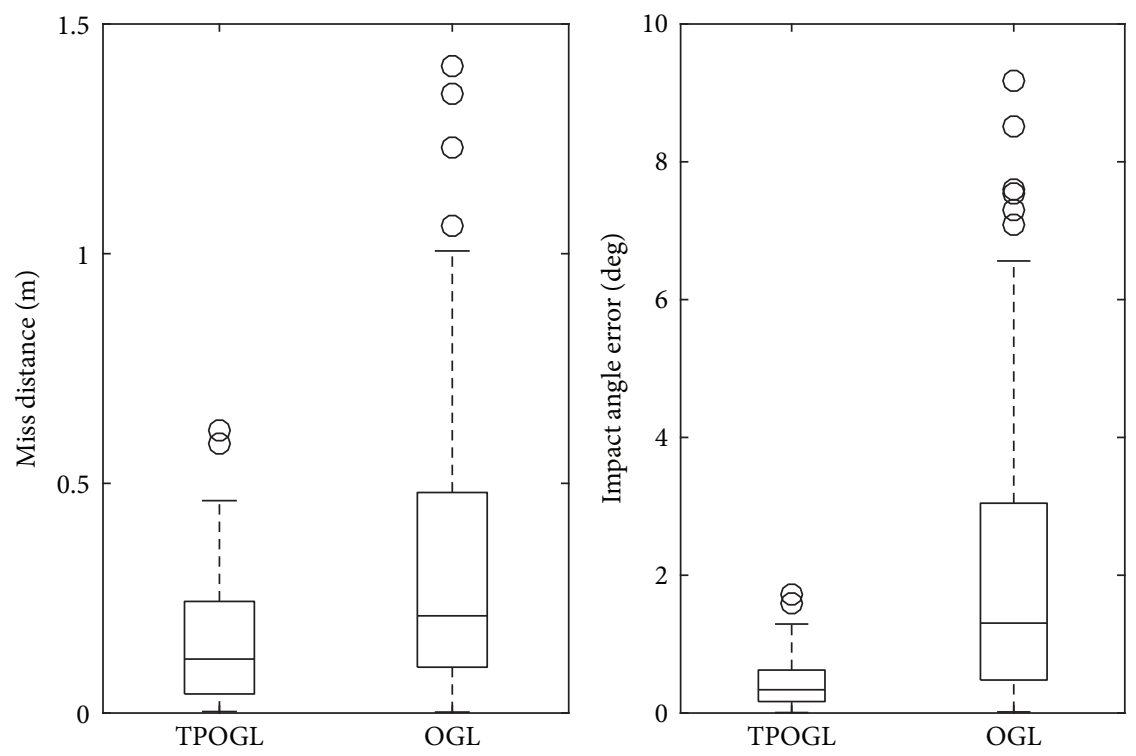

FIGURE 8: Distributions of errors against stationary targets.
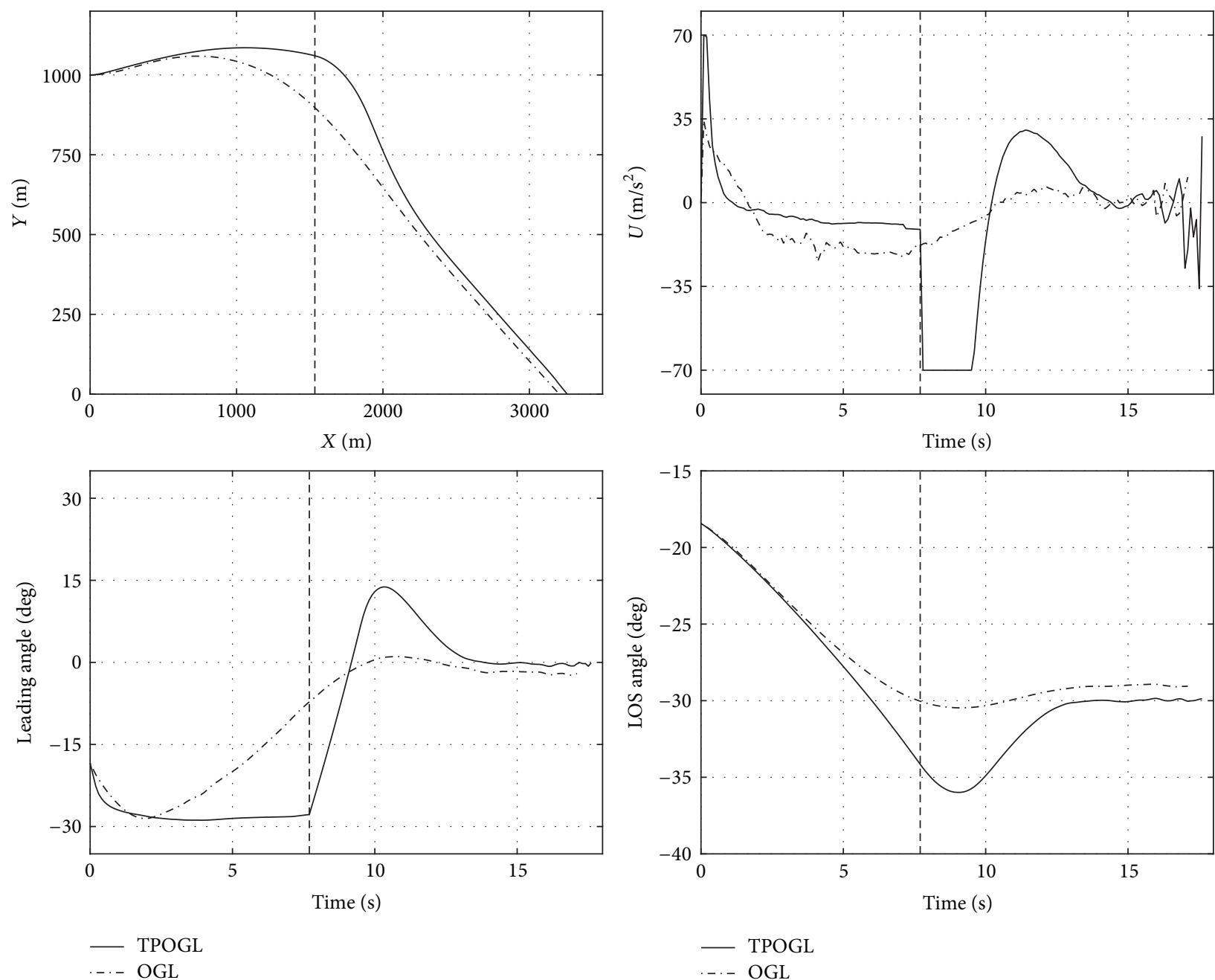

FIGURE 9: Simulation results comparisons against maneuvering targets. 

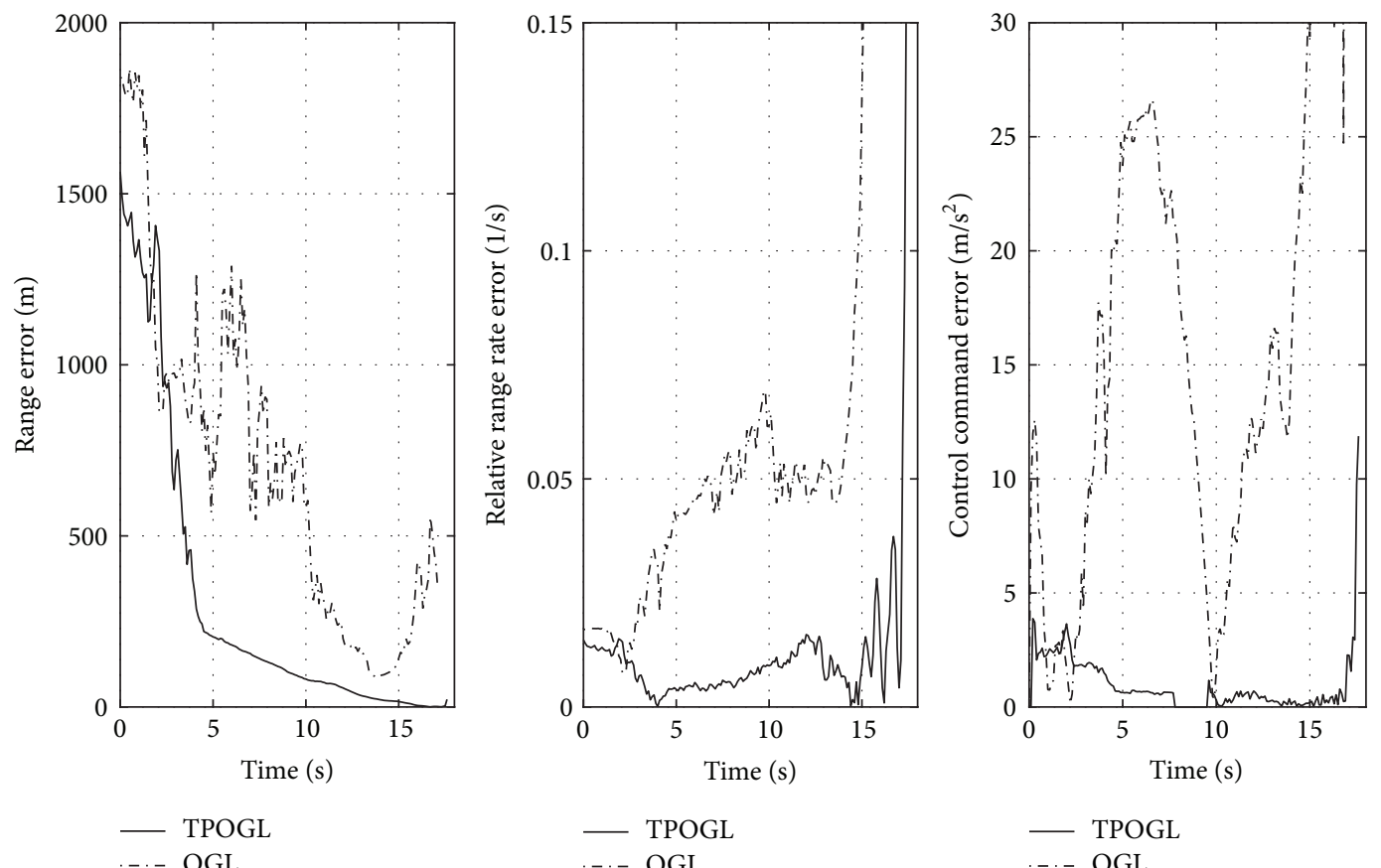

FIGURE 10: Estimation errors comparisons against maneuvering targets.
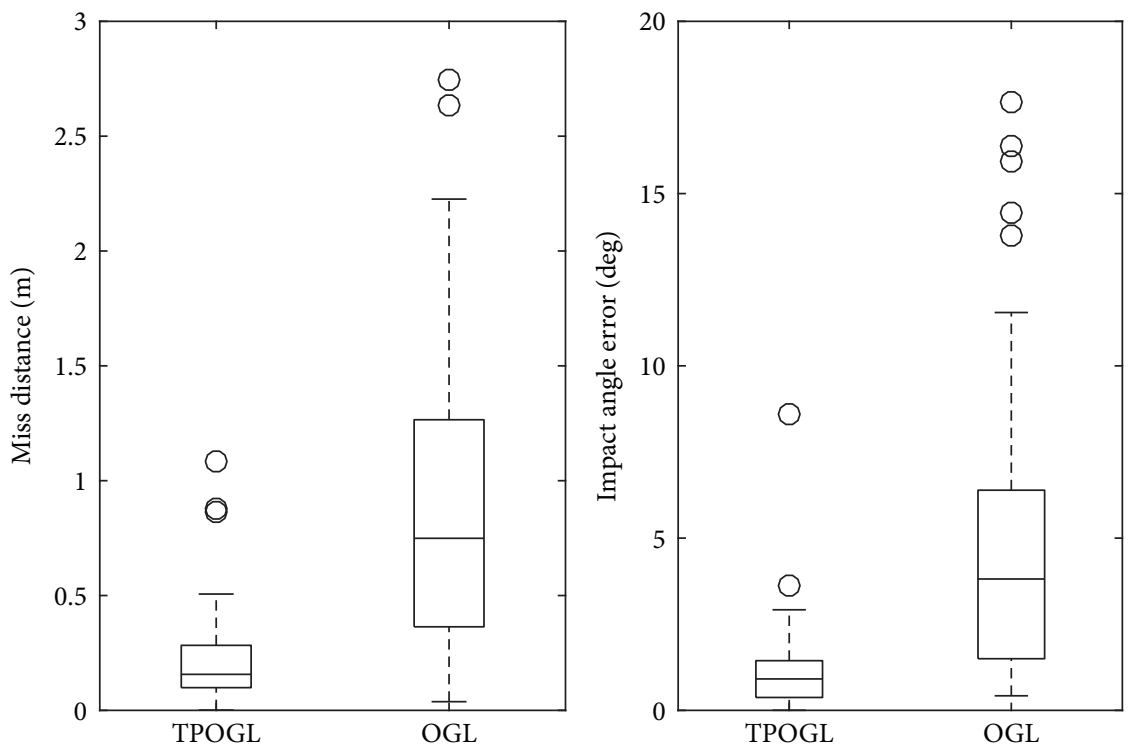

FIGURE 11: Distributions of errors against maneuvering targets.

On the contrary, without considering observability, the errors obtained by OGL in the maneuvering target interception case are much larger than those in the stationary targets interception case.

Similarly, simulations are carried out with a set of 100 Monte Carlo samples with the guidance laws. Each sample differs from the others by measurement noises and target acceleration. The boxplots of miss distances and impact angle errors against maneuvering targets are shown in Figure 11.

From Figure 11, the median value of miss distances obtained by TPOGL is $0.16 \mathrm{~m}$ while that obtained by OGL is $0.75 \mathrm{~m}$, and TPOGL improves the accuracy by $79 \%$. The median value of impact angle errors obtained by TPOGL is $0.91^{\circ}$ while that obtained by OGL is $3.81^{\circ}$, and TPOGL improves the accuracy by $76 \%$. Besides, comparing the results of this case with those of the stationary targets interception case, the target maneuvering has less effect on TPOGL than OGL.

\section{Conclusion}

In this paper, a two-phase optimal guidance law is proposed to improve the estimation accuracy and terminal performances for impact angle constraint engagement. In 
the observing phase, the missile maneuvers to optimize observability by tracking the optimal leading angle using terminal SMC guidance law. In the attacking phase, the missile maneuvers to strike the target with the desired impact angle using optimal guidance law with FOV constraint. Simulation results show that TPOGL can provide better performances including miss distance and impact angle accuracy than the conventional optimal guidance law can. The proposed method has a simple structure and does not require high computational load. Further, the optimal guidance law used in the second phase can be replaced by other impact angle constrained guidance laws as well, which expands the adaptability of the two-phase guidance strategy.

\section{Conflicts of Interest}

The authors declare that there are no conflicts of interest regarding the publication of this paper.

\section{References}

[1] A. Ratnoo and D. Ghose, "Impact angle constrained interception of stationary targets," Journal of Guidance, Control, and Dynamics, vol. 31, no. 6, pp. 1817-1822, 2008.

[2] K. S. Erer and O. Merttopçuoglu, "Indirect impact-anglecontrol against stationary targets using biased pure proportional navigation," Journal of Guidance, Control, and Dynamics, vol. 35, no. 2, pp. 700-703, 2012.

[3] Z. Yang, H. Wang, and D. Lin, "Time-varying biased proportional guidance with seeker's field-of-view limit," International Journal of Aerospace Engineering, vol. 2016, Article ID 9272019, 11 pages, 2016.

[4] C.-K. Ryoo, H. Cho, and M.-J. Tahk, "Time-to-go weighted optimal guidance with impact angle constraints," IEEE Transactions on Control Systems Technology, vol. 14, no. 3, pp. 483-492, 2006.

[5] B.-G. Park, T.-H. Kim, and M.-J. Tahk, "Range-to-go weighted optimal guidance with impact angle constraint and seeker's look angle limits," IEEE Transactions on Aerospace and Electronic Systems, vol. 52, no. 3, pp. 1241-1256, 2016.

[6] S. R. Kumar, S. Rao, and D. Ghose, "Sliding-mode guidance and control for all-aspect interceptors with terminal angle constraints," Journal of Guidance, Control, and Dynamics, vol. 35, no. 4, pp. 1230-1246, 2012.

[7] B. Zhao and J. Zhou, "Smooth Adaptive Finite Time Guidance Law with Impact Angle Constraints," International Journal of Aerospace Engineering, vol. 2016, Article ID 5730168, 2016.

[8] S. He and D. Lin, "A robust impact angle constraint guidance law with seeker's field-of-view limit," Transactions of the Institute of Measurement and Control, vol. 37, no. 3, pp. 317-328, 2015.

[9] V. J. Aidala and S. E. Hammel, "Utilization of Modified Polar Coordinates for Bearings-Only Tracking," IEEE Transactions on Automatic Control, vol. 28, no. 3, pp. 283-294, 1983.

[10] N. J. Gordon, D. J. Salmond, and A. F. M. Smith, "Novel approach to nonlinear/non-gaussian bayesian state estimation," IEE Proceedings F, vol. 140, no. 2, pp. 107-113, 1993.

[11] P. B. Quang, C. Musso, and F. L. Gland, "Particle filtering and the laplace method for target tracking," IEEE Transactions on Aerospace and Electronic Systems, vol. 52, no. 1, pp. 350-366, 2016.
[12] S. C. Nardone, K. F. Gong, and A. G. Lindgren, "Fundamental Properties and Performance of Conventional Bearings-Only Target Motion Analysis," IEEE Transactions on Automatic Control, vol. 29, no. 9, pp. 775-787, 1984.

[13] K. Dogancay, "3D pseudolinear target motion analysis from angle measurements," IEEE Transactions on Signal Processing, vol. 63, no. 6, pp. 1570-1580, 2015.

[14] Y. Oshman and P. Davidson, "Optimization of observer trajectories for bearings-only target localization," IEEE Transactions on Aerospace and Electronic Systems, vol. 35, no. 3, pp. 892-902, 1999.

[15] E. W. Frew, "Observer trajectory generation for target-motion estimation using monocular vision," Diss. Stanford University, 2003.

[16] A. Modirrousta, M. Sohrab, and S. M. M. Dehghan, "A modified guidance law for ground moving target tracking with a class of the fast adaptive second-order sliding mode," Transactions of the Institute of Measurement and Control, vol. 38, no. 7, pp. 819-831, 2016.

[17] S. Battistini and T. Shima, "Differential games missile guidance with bearings-only measurements," IEEE Transactions on Aerospace and Electronic Systems, vol. 50, no. 4, pp. 2906-2915, 2014.

[18] A. J. Sinclair, R. J. Prazenica, and D. E. Jeffcoat, "Optimal and feedback path planning for cooperative attack," Journal of Guidance, Control, and Dynamics, vol. 31, no. 6, pp. 1708-1715, 2008.

[19] S. Lim and H. Bang, "Guidance laws for target localization using vector field approach," IEEE Transactions on Aerospace and Electronic Systems, vol. 50, no. 3, pp. 1991-2003, 2014.

[20] Y. Bar-Shalom, X. Li, and T. Kirubarajan, Estimation with applications to tracking and navigation: theory algorithms and software, John Wiley and Sons, 2004.

[21] S. E. Hammel, P. T. Liu, E. J. Hilliard, and K. F. Gong, "Optimal observer motion for localization with bearing measurements," Computers and Mathematics with Applications, vol. 18, no. 1-3, pp. 171-180, 1989.

[22] W. Lee, H. Bang, and H. Leeghim, "Cooperative localization between small UAVs using a combination of heterogeneous sensor," Aerospace Science and Technology, vol. 27, no. 1, pp. 105111, 2013. 


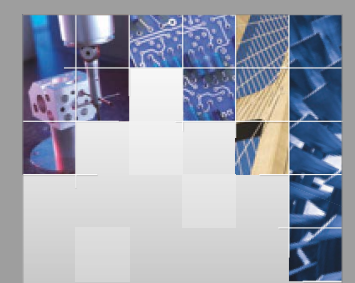

\section{Enfincering}
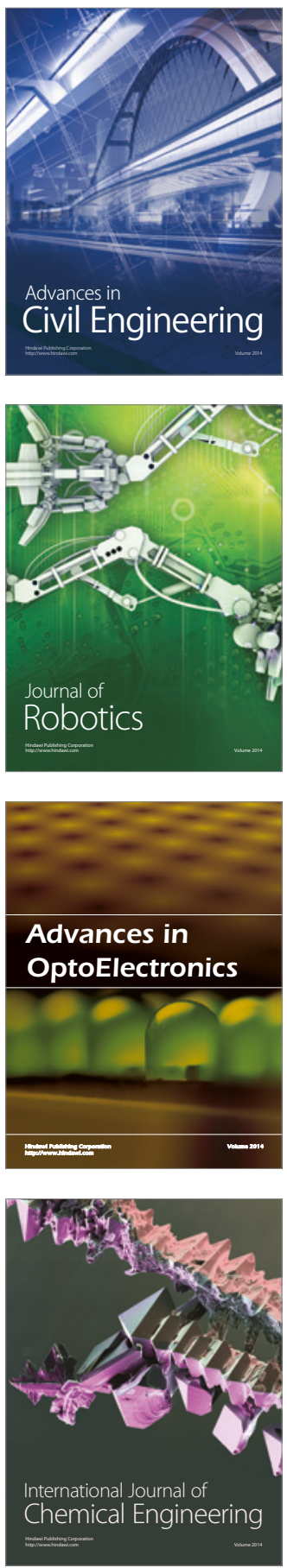

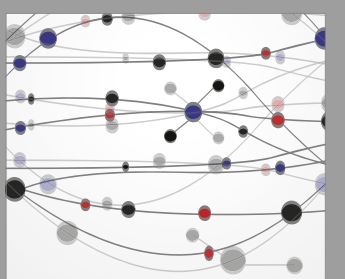

The Scientific World Journal

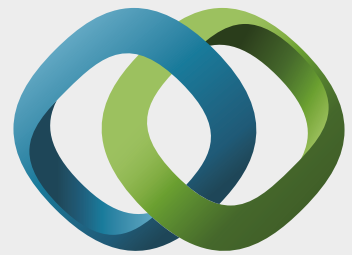

\section{Hindawi}

Submit your manuscripts at

https://www.hindawi.com
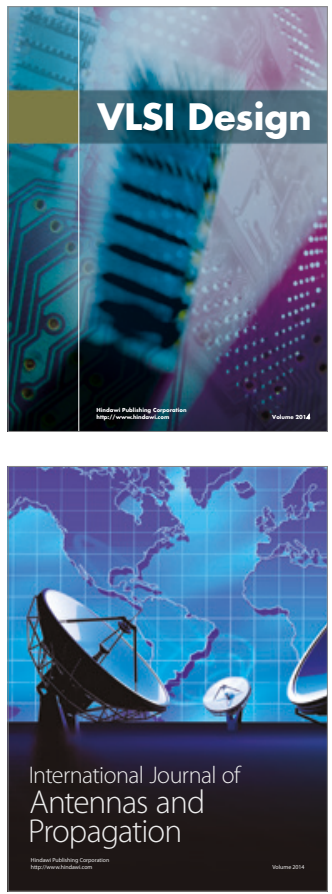

\section{Rotating}

Machinery
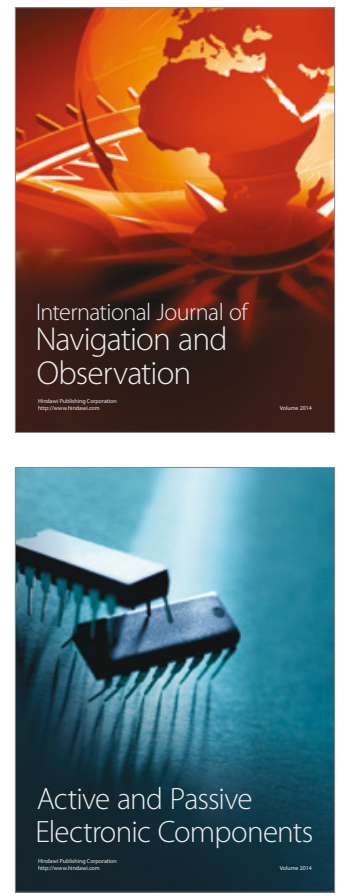
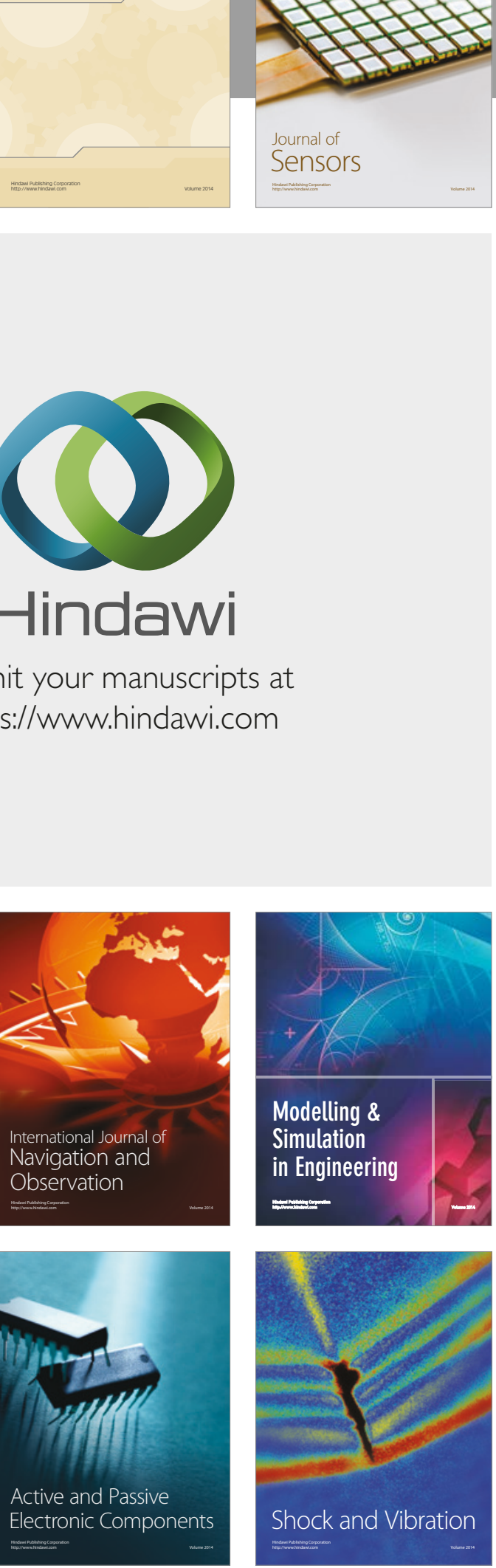
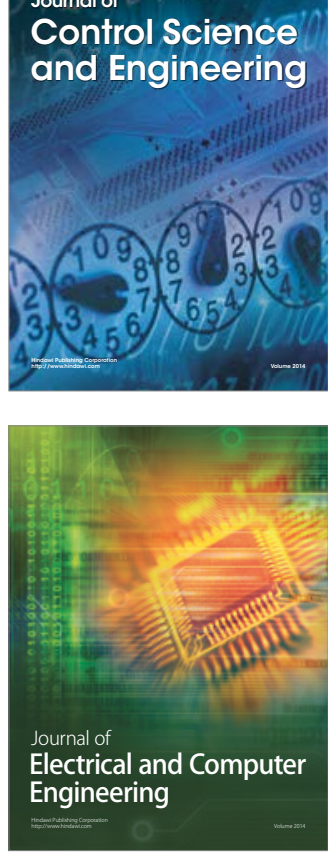

Distributed

Journal of

Control Science

and Engineering
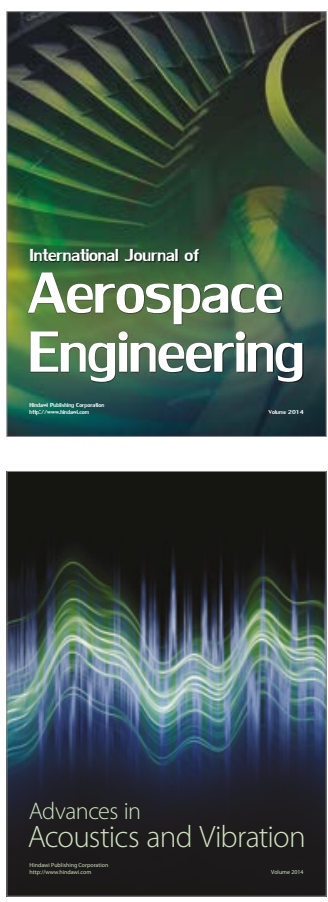

Sensor Networks 